\title{
Shape optimization for the minimum volume of pin fins in simultaneous heat and mass transfer environments
}

\author{
Balaram Kundu • Kwan-Soo Lee
}

Received: 17 May 2011/ Accepted: 17 January 2012/Published online: 1 February 2012

(C) The Author(s) 2012. This article is published with open access at Springerlink.com

\begin{abstract}
A methodology for determining the optimum pin fin profile is introduced to minimize the fin volume for a constrained heat transfer rate under dehumidifying surface conditions. In this methodology, the mass transfer is evaluated using the polynomial variation of humidity ratio with temperature. A scheme is developed for solving the optimum conditions derived as a function of unknown temperature-dependent parameter and tip temperature under both the fully and partially wet surface conditions. The effect of psychrometric properties of the surrounding air on the optimum wet fin profile has been examined. The analysis presented in this study is pertinent to the dry, fully wet, and partially wet surface conditions. In every case study of optimum wet fins, the excess temperature at the tip vanishes with respect to the surrounding temperature. A non-linear temperature distribution in the optimum wet fin has been identified.
\end{abstract}

\section{List of symbols}

$a_{j} \quad$ parameter defined in Eqs. 3 and 4

$A, B, C, D$ constants; see Eq. 2

$b_{j} \quad$ parameters defined in Eqs. 24 and 26

B. Kundu $\cdot$ K.-S. Lee $(\bowtie)$

School of Mechanical Engineering, Hanyang University,

17 Haengdang-dong, Sungdong-gu, Seoul 133-791,

Republic of Korea

e-mail: ksleehy@hanyang.ac.kr

B. Kundu

e-mail: bkundu123@rediffmail.com

B. Kundu

Department of Mechanical Engineering,

Jadavpur University, Kolkata 700 032, India

\begin{tabular}{|c|c|}
\hline $\mathrm{Bi}$ & Biot number, $h y_{b} / k$ \\
\hline$C_{P, \mathrm{a}}$ & $\begin{array}{l}\text { constant pressure specific heat for dry air, } \\
\mathrm{J} \mathrm{kg}^{-1}{ }^{\circ} \mathrm{C}^{-1}\end{array}$ \\
\hline$h$ & $\begin{array}{l}\text { convective heat transfer coefficient, } \\
\mathrm{W} \mathrm{m}{ }^{-2}{ }^{\circ} \mathrm{C}^{-1}\end{array}$ \\
\hline$h_{f g}$ & $\begin{array}{l}\text { latent heat of condensation of moisture, } \\
\mathrm{J} \mathrm{kg}^{-1}\end{array}$ \\
\hline$h_{m}$ & mass transfer coefficient, $\mathrm{kg} \mathrm{m}^{-2} \mathrm{~s}^{-1}$ \\
\hline$k$ & $\begin{array}{l}\text { thermal conductivity of the fin material, } \\
\mathrm{W} \mathrm{m}{ }^{-1}{ }^{\circ} \mathrm{C}^{-1}\end{array}$ \\
\hline$L$ & fin length, $\mathrm{m}$ \\
\hline Le & Lewis number \\
\hline$p_{a}$ & ambient pressure, bar \\
\hline$q$ & actual heat transfer rate, $\mathrm{w}$ \\
\hline$Q$ & $\begin{array}{l}\text { dimensionless actual heat transfer rate; } \\
\text { see Eq. } 11\end{array}$ \\
\hline$T$ & local fin surface temperature, ${ }^{\circ} \mathrm{C}$ \\
\hline$T_{a}$ & ambient temperature, ${ }^{\circ} \mathrm{C}$ \\
\hline$T_{b}$ & base temperature, ${ }^{\circ} \mathrm{C}$ \\
\hline$T_{d}$ & dew-point temperature, ${ }^{\circ} \mathrm{C}$ \\
\hline$T_{0}$ & tip temperature, ${ }^{\circ} \mathrm{C}$ \\
\hline$U$ & $\begin{array}{l}\text { dimensionless fin volume; see Eqs. } 15 a, 15 b \\
\text { and } 32\end{array}$ \\
\hline$V$ & fin volume, $\mathrm{m}^{3}$ \\
\hline$x$ & coordinate shown in Fig. $1, \mathrm{~m}$ \\
\hline$X$ & dimensionless coordinate, $x / L$ \\
\hline$x_{d}$ & $\begin{array}{l}\text { length of the dry region for a partially wet fin } \\
\text { measured from the fin tip, m }\end{array}$ \\
\hline$X_{d}$ & dimensionless length of the dry region, $x_{d} / L$ \\
\hline$y$ & local radius of pin fins, $\mathrm{m}$ \\
\hline$Y$ & dimensionless radius, $y / y_{b}$ \\
\hline$Y^{*}$ & dimensionless local fin thickness, $y / L$ \\
\hline$y_{b}$ & base radius, $\mathrm{m}$ \\
\hline$Z_{O}$ & fin parameter, $\sqrt{\mathrm{Bi}} / \psi$ \\
\hline
\end{tabular}




\section{Greek letters}

$\mu \quad$ parameter; see Eqs. 7 and 22a, 22b

$\phi \quad$ relative humidity of surrounding air

$\Phi(\theta) \quad$ function; see Eqs. 18 and 35

$\theta$ dimensionless local fin surface temperature, $\left(T_{a}-T\right) /\left(T_{a}-T_{b}\right)$

$\theta_{0} \quad$ dimensionless tip temperature, $\left(T_{a}-T_{0}\right) /\left(T_{a}-T_{b}\right)$

$\theta_{d} \quad$ dimensionless dew-point temperature, $\left(T_{a}-T_{d}\right) /\left(T_{a}-T_{b}\right)$

$\omega \quad$ specific humidity of the saturated air, $\mathrm{kg}$ of water vapor per $\mathrm{kg}$ of dry air

$\omega_{a} \quad$ specific humidity of surrounding air, $\mathrm{kg}$ of water vapor per $\mathrm{kg}$ of dry air

$\xi \quad$ dehumidification parameter, $h_{f g} /\left(C_{p, a} \mathrm{Le}^{2 / 3}\right),{ }^{\circ} \mathrm{C}$

$\psi \quad$ dimensionless base thickness, $y_{b} / L$

$\Omega(\theta) \quad$ notation; see Eq. 36

\section{Subscript}

opt optimum

\section{Introduction}

In refrigeration, air conditioning, and aerospace applications, fins are employed to enhance the heat transfer rate between the fin surfaces and the surrounding air that is being cooled. In the aforementioned applications, the fin surface temperature is always lower than the ambient temperature, and the fin surface may be dry, fully wet, or partially wet, depending upon the base temperature, tip temperature, and dew-point temperature of the surrounding air. The temperature at the fin base can be specified according to the application of the design. The dew-point temperature of the surrounding air is solely a function of the psychrometric properties of the air. The fin tip temperature depends primarily upon the thermo-geometric parameters included in the design process. When the relative humidity is very low, a dry surface can be maintained. However, wet surfaces (partially or fully) are commonly

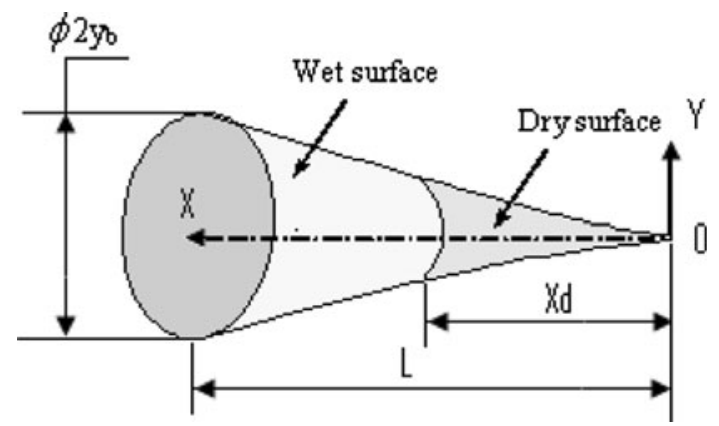

Fig. 1 Schematic of an optimum pin fin under partially wet conditions found for the practical range of relative humidities of the surrounding air.

In many practical applications, heat transfer through extended surfaces occurs under two-phase flow conditions. For example, when humid air encounters the surface of a cooling coil whose temperature is lower than the dew-point temperature, condensation of moisture will take place. Extensive research [1-9] has already been undertaken to analyze the effect of condensation on the performance of rectangular geometric longitudinal fins. It is important to note that in each of these studies, a suitable fin geometry (rectangular, trapezoidal, triangular, etc.) was selected prior to the analysis. However, for a combined heat and mass transfer environment, the mathematical formulation for analyzing the overall performance of a wet fin becomes complex. Utilizing a modified dry fin formula, Threlkeld [2] and McQuiston [3] separately calculated the onedimensional fin efficiency of a rectangular longitudinal fin under fully wet surface conditions. Kilic and Onat [4] employed a quasi-linear one-dimensional model to determine the performance of vertical rectangular wet fins. Coney et al. [5] numerically investigated the performance of vertical rectangular fins subject to condensation of moisture in humid air. An analytical approach to estimating the efficiency of longitudinal straight fins under dry, fully wet, and partially wet surface conditions was intricately devised by $\mathrm{Wu}$ and Bong [6]. Kundu [7] used a decomposition method to carry out a thermal analysis of straight fins under fully wet and partially wet conditions, assuming the humidity ratio of saturated air to be a polynomial function of the fin temperature. Lin et al. [8] experimentally investigated the performance of a rectangular fin in both dry and wet conditions. The thermal analysis and optimization of longitudinal and pin fins of uniform thickness subject to fully wet, partially wet and fully dry surface conditions were carried out analytically by Kundu [9].

It is fact that the heat conduction rate in a fin increases toward the fin base under dehumidifying conditions. Increasing heat conduction in the direction of flow requires an incremented cross-section for better utilization of fin material. Much research has been devoted to the study of wet fins, particularly fins with a variety of tapered profiles, including step reduction in local cross section [10, 11], trapezoidal [12, 13], triangular [14, 15], and convex parabolic [16]. It can be demonstrated (all other conditions being equal) that these complex-profile fins are lighter in weight than flat fins. Nevertheless, they are not the "lightest" fins.

In many industrial applications, such as those pertaining to aerospace, air conditioning, electronic components, automobile radiators, and heat exchangers in vessels, the weight or the available space is a major design consideration. However, the attachment of fins to the primary surface always increases the weight of the system. Therefore, 
in fin design problems, especially in the above applications, it is extremely important to carry out an optimization study to determine the minimum fin volume. There are two approaches available for the optimization analysis of a fin. In the first approach, the shape of the fin is determined that either maximizes the heat transfer rate for a given fin volume or minimizes the fin volume for a given heat transfer rate. In the second approach, the optimum dimensions of a fin with a given profile (rectangular, triangular, etc.) are determined from the optimality criteria. The profile obtained from the first approach is superior in terms of the heat transfer rate per unit volume, and thus the resulting fin shape may be suitable for applications where space and weight constraints are given priority.

Thorough analyses for determining the optimum fin shapes in convective, convective-radiative, volumetric heat generation, and variable convective heat transfer coefficient environments have been carried out by a number of researchers [17-21]. However, under dehumidifying conditions on the fin surface, heat transfer is accompanied by mass transfer, which makes the governing equation for the fins complicated to solve. In order to conduct an analytical analysis, most researchers have adopted a linear relationship between the humidity ratio and the fin surface temperature to calculate the heat transfer through wet fins. The overall performance for fins of a given shape has also been determined. Kundu [22] developed a model for the optimum shape of three common fin geometries under dehumidifying conditions, assuming a linear model. With this assumption, the nature of the governing equation for wet fins does not vary from that of the equation under dry conditions. Hence, the published solution for wet fins is similar to the solution for convective fins. Actually, the saturated air covering the fin surface results from the dehumidification of air. The difference between the humidity ratio of the incoming air being cooled and the air covering the fin surface is the driving force for the mass transfer, which is mainly responsible for making the energy equation nonlinear (if the actual motive force is taken into account). No analysis based on these actual conditions has hitherto been undertaken to determine the optimum profile fins. However, it is important to note that a more precise optimum profile may be extremely important in mobile systems, especially in aircraft applications, where additional weight is always a critical issue. Up to now, researchers have given little attention to analytical procedures for determining the minimum weight of a pin-fin geometry under wet conditions.

The present work focuses on determining an optimum shape for pin fins subject to combined heat and mass transfer. Since the humidity ratio of saturated air is a function of temperature alone, it can be represented by a third-degree polynomial using regression analysis. Based on this fact, the governing equation for wet pin fins is derived. The temperature distribution functions for both fully and partially wet fins are assumed to follow a power law. In this research, the optimization is carried out for a constant heat transfer duty. The objective function (fin volume) is formulated in terms of a known design constant. The principle of minimizing the fin volume is adopted to establish an optimality criterion. Finally, the optimum fin profile and temperature distribution for an optimum profile fin are determined.

\section{Analysis}

Simultaneous heat and mass transfer takes place on a fin surface when the surface temperature is lower than the dew-point temperature of the surrounding air, and moisture in the air is condensed. During this process, the incoming air adjacent to the fin surface is saturated according to the fin surface temperature. The fin surface is always possible to find different surface conditions: fully wet, partially wet, and dry [9]. Analyses of dry surface fins can be found in the literature. In the present study, separate analyses of fully and partially wet fins are carried out in the following subsections. However, the analysis of dry surface fins can be obtained from the analysis of the fully wet fin by setting only the latent heat of condensation value equal to zero.

\subsection{Fully wet}

Under steady-state conditions, the governing equation for pin fins subject to combined heat and mass transfer can be written as follows $[6,23]$ :

$$
\begin{aligned}
& \frac{d}{d x}\left(y^{2} \frac{d T}{d x}\right)-\frac{h y}{k}\left[\left(T-T_{a}\right)+\frac{h_{m}}{h}\left(\omega-\omega_{a}\right) h_{f g}\right] \\
& \quad \times \sqrt{1+\left(\frac{d y}{d x}\right)^{2}}=0
\end{aligned}
$$

It may not be possible to solve Eq. 1 analytically unless the relationship between $T$ and $\omega$ is known. However, since the fin surface is covered with saturated air, the relationship between $T$ and $\omega$ follows the saturation curve in the psychrometric chart. This curve can be represented as a function of the humidity ratio and the fin surface temperature. Using the best-fit method, the humidity ratio for the saturation curve can be expressed as a cubic polynomial [7]:

$\omega=A+B T+C T^{2}+D T^{3}$

where the constants $A, B, C$, and $D$ are $3.7444 \times 10^{-3}$, $0.3078 \times 10^{-3}, \quad 0.0046 \times 10^{-3}$, and $0.0004 \times 10^{-3}$, respectively. 
Combining Eqs. 1 and 2, the governing equation can be written in dimensionless form by using the Chilton-Colburn analogy and the thin-fin assumption:

$\frac{d}{d X}\left(Y^{2} \frac{d \theta}{d X}\right)-Z_{0}^{2} Y \sum_{j=1}^{4} a_{j} \theta^{j-1}=0$

where
The actual heat transfer rate $q$ can be derived from Fourier's law of heat conduction applied at the fin base:

$Q=\frac{q h}{\pi k^{2}\left(T_{a}-T_{b}\right)}=\operatorname{Bi} \psi\left(Y^{2} \frac{d \theta}{d X}\right)_{X=1}$

Combining Eqs. 8 and 11, the following expression for the heat transfer rate is obtained:

$\left(\begin{array}{ll}a_{1} & a_{2} \\ a_{3} & a_{4}\end{array}\right)=\left(\begin{array}{cc}\xi\left(\omega_{a}-A-B T_{a}-C T_{a}^{2}-D T_{a}^{3}\right)-\left(T_{a}-T_{b}\right) & 1+B \xi+2 C \xi T_{a}+3 D \xi T_{a}^{2} \\ -\xi\left(T_{a}-T_{b}\right)\left(C+3 D T_{a}\right) & D \xi\left(T_{a}-T_{b}\right)^{2}\end{array}\right)$

and

$$
\begin{aligned}
\left(\begin{array}{llll}
B i & \psi & Z_{0} & \theta
\end{array}\right) \\
\quad=\left(\begin{array}{llll}
h y_{b} / k & y_{b} / L & \sqrt{B i} / \psi & \left(T_{a}-T\right) /\left(T_{a}-T_{b}\right)
\end{array}\right)
\end{aligned}
$$

Equation 3 is solved in conjunction with the following boundary conditions:

$Y^{2} \frac{d \theta}{d X}=0 \quad$ at $\quad X=0$

$\theta=1 \quad$ at $\quad X=1$

It is now assumed that the fin profile can be correlated with the local fin temperature via a function of the form

$Y=\theta^{\mu}$ for $\quad \mu>0$

Multiplying Eq. 3 by $Y^{2}(d \theta / d X)$ and then integrating using Eqs. $6 \mathrm{~b}$ and 7 yields

$\left(Y^{2} \frac{d \theta}{d X}\right)=2^{1 / 2} Z_{0}\left[\sum_{n=1}^{4} \frac{a_{n}}{n+3 \mu}\left(\theta^{n+3 \mu}-\theta_{0}^{n+3 \mu}\right)\right]^{1 / 2}$

where $\theta_{0}$ is the dimensionless tip temperature.

Combining Eqs. 7 and 8, the temperature distribution in the fin is expressed as a function of $X$ :

$\int_{\theta=\theta_{0}}^{\theta} \frac{\theta^{2 \mu} d \theta}{\left[\sum_{n=1}^{4} \frac{a_{n}}{n+3 \mu}\left(\theta^{n+3 \mu}-\theta_{0}^{n+3 \mu}\right)\right]^{1 / 2}}=2^{1 / 2} Z_{0} \int_{X=0}^{X} d X$

The fin parameter $Z_{0}$ can be determined by using Eqs. $6 \mathrm{~b}$ and 9:

$Z_{0}=2^{-1 / 2} \int_{\theta=\theta_{0}}^{1} \frac{\theta^{2 \mu} d \theta}{\left[\sum_{n=1}^{4} \frac{a_{n}}{n+3 \mu}\left(\theta^{n+3 \mu}-\theta_{0}^{n+3 \mu}\right)\right]^{1 / 2}}$
$Q=2^{1 / 2} Z_{0}^{3} \psi^{3}\left[\sum_{n=1}^{4} \frac{a_{n}}{n+3 \mu}\left(1-\theta_{0}^{n+3 \mu}\right)\right]^{1 / 2}$

With a constrained heat transfer rate, the ratio of the base thickness to the length $\psi$ can be explicitly written as follows:

$\psi=\frac{Q^{1 / 3} 2^{1 / 3}\left[\sum_{n=1}^{4} \frac{a_{n}}{n+3 \mu}\left(1-\theta_{0}^{n+3 \mu}\right)\right]^{-1 / 6}}{\int_{\theta=\theta_{0}}^{1} \frac{\theta^{2 \mu} d \theta}{\left[\sum_{n=1}^{4} \frac{a_{n}}{n+3 \mu}\left(\theta^{n+3 \mu}-\theta_{0}^{n+3 \mu}\right)\right]^{1 / 2}}}$

The volume of the fin is expressed in dimensionless form as

$U=\frac{1}{\pi} \frac{h^{3} V}{k^{3}}=\psi^{5} Z_{0}^{6} \int_{X=0}^{1} Y^{2} d X$

Eq. 15a can be written by using Eqs. 7 and 9 as

$U=2^{-1 / 2} \psi^{5} Z_{0}^{5} \int_{\theta=\theta_{0}}^{1} \frac{\theta^{4 \mu} d \theta}{\left[\sum_{n=1}^{4} \frac{a_{n}}{n+3 \mu}\left(\theta^{n+3 \mu}-\theta_{0}^{n+3 \mu}\right)\right]^{1 / 2}}$

From the above equation, it can be inferred that the fin volume is a function of $\psi, Z_{0}, \theta_{0}$, and $\mu$. The thermogeometric parameters $Z_{0}$ and $\psi$ are also functions of $\theta_{0}$ and $\mu$, given by Eqs. 10 and 14, respectively. Therefore, the dimensionless fin volume is dependent upon $\theta_{0}$ and $\mu$. The optimum conditions for a constrained heat transfer rate can be derived by minimizing the fin volume for a parametric variation of $\mu$ and $\theta_{0}$. From the minimization principle, the optimality conditions can be expressed mathematically as follows: 


$$
\begin{aligned}
& 5\left(Z_{0} \frac{\partial \psi}{\partial \mu}+\psi \frac{\partial Z_{0}}{\partial \mu}\right) \int_{\theta=\theta_{0}}^{1} \frac{\theta^{4 \mu} d \theta}{[\Phi(\theta)]^{1 / 2}} \\
& +Z_{0} \psi \int_{\theta=\theta_{0}}^{1}\left\{4 \theta^{4 \mu}[\Phi(\theta)]^{-1 / 2} \ln \theta-\frac{1}{2}[\Phi(\theta)]^{-3 / 2} \theta^{4 \mu} \frac{\partial \Phi(\theta)}{\partial \mu}\right\} \\
& d \theta=0
\end{aligned}
$$

and

$$
\begin{aligned}
& 5\left(Z_{0} \frac{\partial \psi}{\partial \theta_{0}}+\psi \frac{\partial Z_{0}}{\partial \theta_{0}}\right) \int_{\theta=\theta_{0}}^{1} \frac{\theta^{4 \mu} d \theta}{[\Phi(\theta)]^{1 / 2}} \\
& \quad-\frac{Z_{0} \psi}{2} \int_{\theta=\theta_{0}}^{1}[\Phi(\theta)]^{-3 / 2} \theta^{4 \mu} \frac{\partial \Phi(\theta)}{\partial \theta_{0}} d \theta-\frac{Z_{0} \psi \theta_{0}^{4 \mu}}{\left[\Phi\left(\theta_{0}^{+}\right)\right]^{1 / 2}}=0
\end{aligned}
$$

where

$\Phi(\theta)=\sum_{n=1}^{4} \frac{a_{n}}{n+3 \mu}\left(\theta^{n+3 \mu}-\theta_{0}^{n+3 \mu}\right)$

and the value $\theta_{0}^{+}$which is taken a very closer to the $\theta_{0}$ to avoid the singularity of the function $\Phi$. In order to determine the optimum values of $\mu$ and $\theta_{0}$, it may be necessary to solve Eqs. 16 and 17 simultaneously. The Newton-Raphson iterative method [24] for determining multiple roots can be employed for the solution. The partial derivatives $\partial Z_{0} / \partial \mu, \partial Z_{0} / \partial \theta_{0}, \partial \psi / \partial \mu, \partial \psi / \partial \theta_{0}$, $\partial^{2} Z_{0} / \partial \mu^{2}, \partial^{2} Z_{0} / \partial \theta_{0}^{2}, \partial^{2} \psi / \partial \mu^{2}, \partial^{2} \psi / \partial \theta_{0}^{2}, \partial^{2} Z_{0} / \partial \mu \partial \theta_{0}$, and $\partial^{2} \psi / \partial \mu \partial \theta_{0}$ are utilized in the solution process, and can be determined by successive differentiation of Eqs. 10 and 14 with respect to $\mu$ and $\theta_{0}$ separately. Integration of Eqs. 16 and 17 is performed numerically using Simpson's $1 / 3$ rule. In this connection, it should be noted that the initial values of $\mu$ and $\theta_{0}$ used in the calculation must be reasonably close to the optimum values, or else the solution may diverge. After the optimum $\mu$ and $\theta_{0}$ have been obtained, the optimum design parameters (fin volume, $Z_{0}$, and $\psi$ ) can be determined for given thermo-physical and psychrometric design conditions. The fin profile and the temperature distribution can also be estimated under the optimum conditions.

\subsection{Partially wet}

Owing to variations in the psychrometric conditions of the surrounding air, a fin surface may become partially wet. The energy equation of a partially wet fin can be constructed by writing separate energy balance equations for dry and wet surfaces as follows:

$$
\begin{aligned}
& \frac{d}{d X}\left(Y^{2} \frac{d \theta}{d X}\right)-Z_{0}^{2} Y \theta=0 \\
& \quad \text { dry surface }\left(0 \leq X \leq X_{d}\right) \text { and } X_{d}>0
\end{aligned}
$$

and

$\frac{d}{d X}\left(Y^{2} \frac{d \theta}{d X}\right)-Z_{0}^{2} Y \sum_{j=1}^{4} a_{j} \theta^{j-1}=0$

wet surface $\left(X_{d} \leq X \leq 1\right)$ and $X_{d}>0$

The fin surface temperature decreases from tip to base when the base temperature is maintained at a constant value lower than the surrounding temperature. Thus, the fin surface temperature may equal the dew-point temperature at some distance $X_{d}$ from the tip, and the dry and wet fin sections intersect at that point. Continuity of temperature and heat flux can be assumed at the junction of the dry and wet sections. Therefore, Eqs. 19a, 19b is subject to the following boundary conditions:

$Y^{2} \frac{d \theta}{d X}=0 \quad$ at $\quad X=0$

$\left(\frac{d \theta}{d X}\right)_{X=X_{d}^{-}}=\left(\frac{d \theta}{d X}\right)_{X=X_{d}^{+}} \quad$ at $\quad X=X_{d}$

$\theta=\theta_{d} \quad$ at $\quad X=X_{d}$

and

$\theta=1 \quad$ at $\quad X=1$

Multiplying Eqs. 19a, 19b by $Y^{2} d \theta / d X$ and then integrating using boundary condition 20a yields

$$
\left(Y^{2} \frac{d \theta}{d X}\right)^{2}=2 Z_{0}^{2} \int_{X=0}^{X} Y^{3} \theta d \theta \quad\left(0 \leq X \leq X_{d}\right)
$$

and

$$
\begin{gathered}
\left(Y^{2} \frac{d \theta}{d X}\right)^{2}=\left(Y^{2} \frac{d \theta}{d X}\right)_{X=X_{d}}^{2}+2 Z_{0}^{2} \int_{X=X_{d}}^{X} \sum_{j=1}^{4} a_{j} \theta^{j-1} Y^{3} d \theta \\
\left(X_{d} \leq X \leq 1\right)
\end{gathered}
$$

The temperature distribution in the dry and wet sections is assumed to have the form of a power function satisfying temperature boundary conditions:

$Y=Y_{d} \frac{\theta^{\mu}}{\theta_{d}^{\mu}} \quad\left(0 \leq X \leq X_{d}\right)$

and

$Y=\frac{Y_{d}-\theta_{d}^{\mu}+\left(1-Y_{d}\right) \theta^{\mu}}{\left(1-\theta_{d}^{\mu}\right)} \quad\left(X_{d} \leq X \leq 1\right)$

After being integrated, Eqs. 21a, 21b can be rewritten as follows, using Eqs. 22a, 22b: 


$$
\left(Y^{2} \frac{d \theta}{d X}\right)^{2}=\left(\frac{2}{2+3 \mu}\right) Z_{0}^{2} \frac{Y_{d}^{3}}{\theta_{d}^{3 \mu}}\left(\theta^{2+3 \mu}-\theta_{0}^{2+3 \mu}\right)
$$

and

$$
\begin{aligned}
\left(Y^{2} \frac{d \theta}{d X}\right)^{2}= & \left(\frac{2}{2+3 \mu}\right) Z_{0}^{2} \frac{Y_{d}^{3}}{\theta_{d}^{3 \mu}}\left(\theta_{d}^{2+3 \mu}-\theta_{0}^{2+3 \mu}\right) \\
& +2 Z_{0}^{2} \sum_{n=1}^{4} b_{n} \sum_{j=1}^{4} \frac{a_{j}}{j+3 \mu}\left(\theta^{j+3 \mu}-\theta_{d}^{j+3 \mu}\right)
\end{aligned}
$$

and

$X_{d}=\frac{(2+3 \mu)^{1 / 2}}{2^{1 / 2} Z_{0}} \int_{\theta=\theta_{0}}^{\theta_{d}} \frac{\theta^{2 \mu} d \theta}{\left(\theta^{2+3 \mu}-\theta_{0}^{2+3 \mu}\right)^{1 / 2}}$

The geometrical parameter $\psi$ can be estimated for a fin with constrained heat transfer rate $Q$ :

where

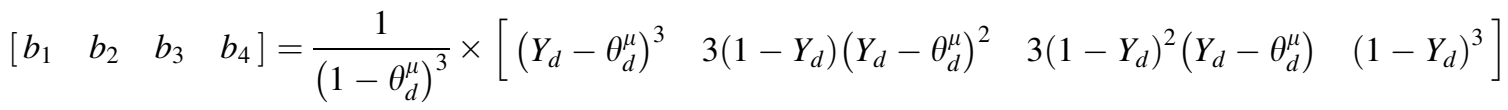

Combining Eqs. 20b, 22a and 22b, the semi-finthickness at the junction of the dry and wet sections is given by

$Y_{d}=\theta_{d}^{\mu}$

From Eq. 25, it can be inferred that the dry length depends solely upon the dew-point temperature of the surrounding air. The fin surface becomes partially wet if the relative humidity of the surrounding air is less than $100 \%$. Equation 24 can be simplified by using Eq. 25:

$\left(\begin{array}{llll}b_{1} & b_{2} & b_{3} & b_{4}\end{array}\right)=\left(\begin{array}{llll}0 & 0 & 0 & 1\end{array}\right)$

Equation 23 b can be rewritten using Eq. 24 as

$$
\begin{aligned}
& \left(Y^{2} \frac{d \theta}{d X}\right)=2^{1 / 2} Z_{0} \\
& \quad \times\left[\frac{\left(\theta_{d}^{2+3 \mu}-\theta_{0}^{2+3 \mu}\right)}{2+3 \mu}+\sum_{j=1}^{4} \frac{a_{j}}{j+3 \mu}\left(\theta^{j+3 \mu}-\theta_{d}^{j+3 \mu}\right)\right]^{1 / 2}
\end{aligned}
$$

The fin parameter $Z_{0}$ and the dry length $X_{d}$ can be obtained from Eqs. 20d and 23a, 23b:

$$
\begin{aligned}
Z_{0}= & \frac{(2+3 \mu)^{1 / 2}}{2^{1 / 2}} \int_{\theta=\theta_{0}}^{\theta_{d}} \frac{\theta^{2 \mu} d \theta}{\left(\theta^{2+3 \mu}-\theta_{0}^{2+3 \mu}\right)^{1 / 2}} \\
& +\frac{1}{2^{1 / 2}} \int_{\theta=\theta_{d}}^{1} \frac{\theta^{2 \mu} d \theta}{\left[\frac{\left(\theta_{d}^{2+3 \mu}-\theta_{0}^{2+3 \mu}\right)}{2+3 \mu}+\sum_{j=1}^{4} \frac{a_{j}\left(\theta^{2+3 \mu}-\theta_{d}^{2+3 \mu}\right)}{j+3 \mu}\right]^{1 / 2}}
\end{aligned}
$$

$$
\psi=\frac{Q^{1 / 3} 2^{-1 / 6}}{Z_{0}}\left[\frac{\theta_{d}^{2+3 \mu}-\theta_{0}^{2+3 \mu}}{2+3 \mu}+\sum_{j=1}^{4} \frac{a_{j}\left(1-\theta_{d}^{j+3 \mu}\right)}{j+3 \mu}\right]^{-1 / 6}
$$

The fin volume of a partially wet fin can be calculated from the following expression:

$U=\frac{1}{\pi} \frac{h^{3} V}{k^{3}}=Z_{0}^{6} \psi^{5}\left[\int_{X=0}^{X_{d}} Y^{2} d X+\int_{X=X_{d}}^{1} Y^{2} d X\right]$

Equation 31 is now integrated by employing Eqs. 23a, 23b:

$$
\begin{aligned}
U= & \frac{Z_{0}^{5} \psi^{5}}{2^{1 / 2}}\left[(2+3 \mu)^{1 / 2} \int_{\theta=\theta_{0}}^{\theta_{d}} \frac{\theta^{4 \mu} d \theta}{\left(\theta^{2+3 \mu}-\theta_{0}^{2+3 \mu}\right)^{1 / 2}}\right. \\
& \left.+\int_{\theta=\theta_{d}}^{1} \frac{\theta^{4 \mu} d \theta}{\left\{\frac{\theta_{d}^{2+3 \mu}-\theta_{0}^{2+3 \mu}}{2+3 \mu}+\sum_{j=1}^{4} \frac{a_{j}\left(\theta^{j+3 \mu}-\theta_{d}^{j+3 \mu}\right)}{j+3 \mu}\right\}^{1 / 2}}\right]
\end{aligned}
$$

The above expression for fin volume is a function of $\mu$ and $\theta_{0}$ for a given heat transfer rate and thermopsychrometric parameters. The condition for optimality can be derived from the principle of minimization of fin volume. Hence, the optimality criterion is obtained by separately setting the first derivatives of Eq. 32 with respect to $\mu$ and $\theta_{0}$ equal to 0 . This yields 


$$
\begin{aligned}
& 5\left(Z_{0} \frac{\partial \psi}{\partial \mu}+\psi \frac{\partial Z_{0}}{\partial \mu}\right) \\
& \left\{(2+3 \mu)^{1 / 2} \int_{\theta=\theta_{0}}^{\theta_{d}} \Phi(\theta) d \theta+\int_{\theta=\theta_{d}}^{1} \Omega(\theta) d \theta\right\} \\
& +Z_{0} \psi\left[\frac{3}{2}(2+3 \mu)^{-1 / 2} \int_{\theta=\theta_{0}}^{\theta_{d}} \Phi(\theta) d \theta+(2+3 \mu)^{1 / 2}\right. \\
& \left.\quad \times \int_{\theta=\theta_{0}}^{\theta_{d}} \frac{\partial \Phi(\theta)}{\partial \mu} d \theta+\int_{\theta=\theta_{d}}^{1} \frac{\partial \Omega(\theta)}{\partial \mu} d \theta\right]=0
\end{aligned}
$$

and

$$
\begin{aligned}
& 5\left(Z_{0} \frac{\partial \psi}{\partial \theta_{0}}+\psi \frac{\partial Z_{0}}{\partial \theta_{0}}\right) \\
& \quad \times\left[(2+3 \mu)^{1 / 2} \int_{\theta=\theta_{0}}^{\theta_{d}} \Phi(\theta) d \theta+\int_{\theta=\theta_{d}}^{1} \Omega(\theta) d \theta\right] \\
& \quad+Z_{0} \psi\left[(2+3 \mu)^{1 / 2}\left\{\int_{\theta=\theta_{0}}^{\theta_{d}} \frac{\partial \Phi(\theta)}{\partial \theta_{0}} d \theta-\Phi\left(\theta_{0}^{+}\right)\right\}+\int_{\theta=\theta_{d}}^{1} \frac{\partial \Omega(\theta)}{\partial \theta_{0}}\right]=0
\end{aligned}
$$

where

$\Phi(\theta)=\frac{\theta^{4 \mu}}{\left(\theta^{2+3 \mu}-\theta_{0}^{2+3 \mu}\right)^{1 / 2}}$

and

$$
\Omega(\theta)=\frac{\theta^{4 \mu}}{\left[\frac{\left(\theta_{d}^{2+3 \mu}-\theta_{0}^{2+3 \mu}\right)}{2+3 \mu}+\sum_{j=1}^{4} \frac{a_{j}\left(\theta^{j+3 \mu}-\theta_{d}^{j+3 \mu}\right)}{j+3 \mu}\right]^{1 / 2}}
$$

The optimum values of $\mu$ and $\theta_{0}$ are obtained by solving Eqs. 33 and 34 simultaneously. A root-finding algorithm based on the Newton-Raphson method can be used to derive the optimum design conditions.

\section{Results and discussion}

Three psychrometric properties of air are used to generate the results: dry-bulb temperature, humidity ratio, and atmospheric pressure. In a fin design problem, base temperature is also specified. These four properties can be used to determine the optimization study of wet fins. From the mathematical formulation, it is obvious that the optimization criterion depends upon the parameter $\mu$ and the tip temperature $\theta_{0}$. Firstly, the effect of these two parameters on the fin optimization is studied. It should be noted that the present analysis of fully wet fins can be applied to dry surfaces simply by setting the latent heat of condensation equal to 0 . Figure 2 depicts the fin volume as a function of the dimensionless tip temperature for different values of $\mu$ and design conditions. The results for completely dry and fully wet surfaces are plotted in Fig. 2a, b. For values of $\mu<1$ (say $\mu=0.5$ ), a remarkable variation of fin volume with respect to $\theta_{0}$ is observed, irrespective of the surface conditions. The fin volume reaches a minimum at a particular tip temperature, and thus there exists an optimum tip temperature and optimum tip thickness for any specified $\mu$. The dependency of the fin volume on the parameter $\mu$ can also be deduced from Fig. 2. The results indicate that the minimum fin volume occurs at a particular $\mu$ and $\theta_{0}$. Specifically, the smallest fin volume is achieved for the optimum $\mu$ at which the tip temperature $\theta_{0}$ vanishes. A comparison of Fig. 2a, b demonstrates that for a constant heat transfer duty, the nature of the variation of fin volume
Fig. 2 Fin volume as a function of tip temperature and the optimality variable $\mu$ : a dry surface; and $\mathbf{b}$ wet surface
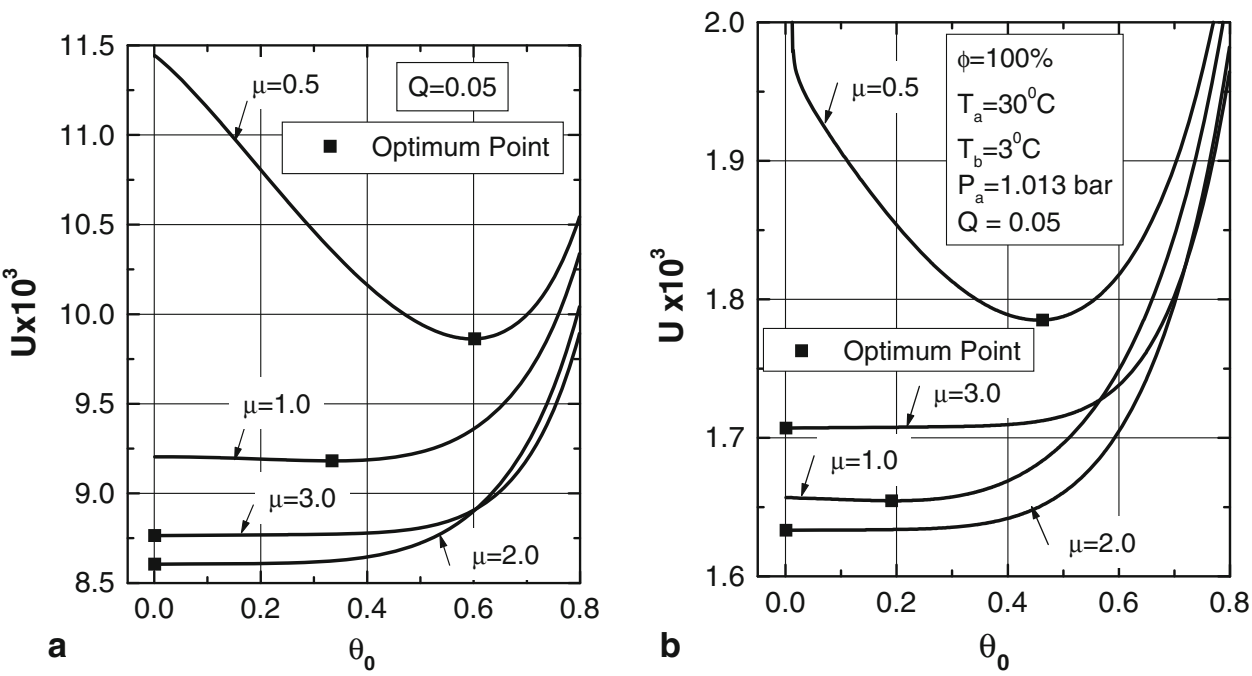
with respect to $\theta_{0}$ is not altered by changes in surface conditions. Nevertheless, the required fin volume is much less in the case of fully wet fins, since more heat is evolved by the latent heat of condensation.

The influence of the optimization parameter $\mu$ on the fin volume is plotted in Fig. 3 for different surface conditions. From the previous figure, it may be inferred that the dimensionless tip temperature vanishes at the optimum point, and hence Fig. 3 and the remaining figures are based on this optimum condition. With a constrained heat transfer rate, the variation of fin volume with respect to $\mu$ has the same nature for dry, fully wet, and partially wet surfaces. The optimum value of $\mu$ is a function of the surface condition in a design problem. The optimum value of $\mu$ for a dry fin is 2.0. The optimum value of $\mu$ for a fully wet fin is 1.501. The optimum value of $\mu$ for partially wet fins lies between 1.501 and 2.0, depending upon the relative humidity of the air determined in the design analysis. It can be inferred from Fig. 3 that as the heat transfer rate
$Q$ increases, the minimum fin volume increases, but the optimum value of $\mu$ does not change. Here it should be noted that using this optimum $\mu$ value for dry fins, the present analysis can be validated easily with the published result [22]. In this study, for the dry surface, both $\xi$ and $\theta_{0}$ are considered zero in the analysis part of the fully wet surface condition. As a result, $a_{1}, a_{3}$ and $a_{4}$ are zero and $a_{2}=1$. With these values, $Z_{0}$ can be determined from Eq. 10, and $Z_{0}=2$ is found. Substituting all aforementioned values in Eq. 9, one can get temperature as a linear function of $X$-coordinate. On the other hand, in the previous work [22], the same expression was evaluated for the pin fin with dry surfaces.

Next, the temperature distribution and fin profile for an optimum fin are shown in Fig. 4 under fully wet surface conditions. Under these conditions, the temperature distribution of the fin is not exactly linear, as in the case of a dry surface fin. As the constraint $Q$ varies, the temperature distribution in the optimum fins is unaltered, as
Fig. 3 Optimum point for fins with respect to fin volume and the optimality variable $\mu$ under various surface conditions: a dry surface; $\mathbf{b}$ fully wet surface; and c partially wet surface
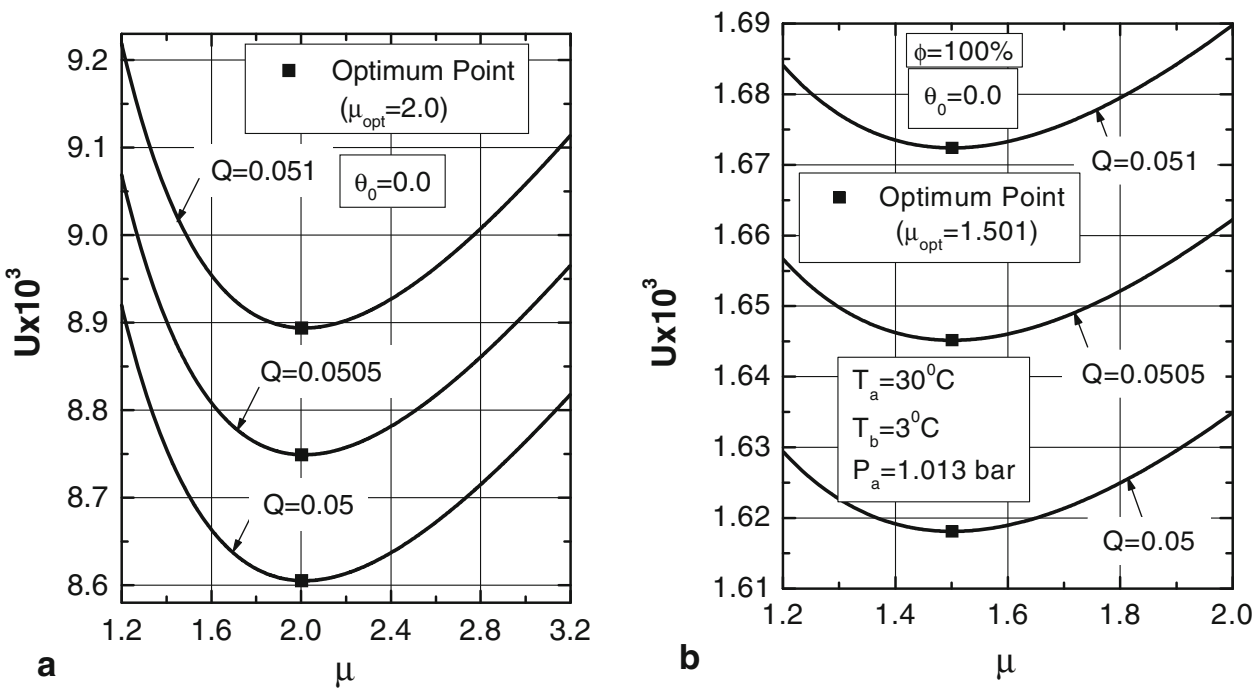
Fig. 4 Dimensionless temperature and fin profiles for optimum fins with fully wet surfaces: a temperature distribution; and $\mathbf{b}$ fin profile


Fig. 5 Dimensionless temperature and fin profiles for optimum fins with partially wet surfaces: a temperature distribution; and $\mathbf{b}$ fin profile

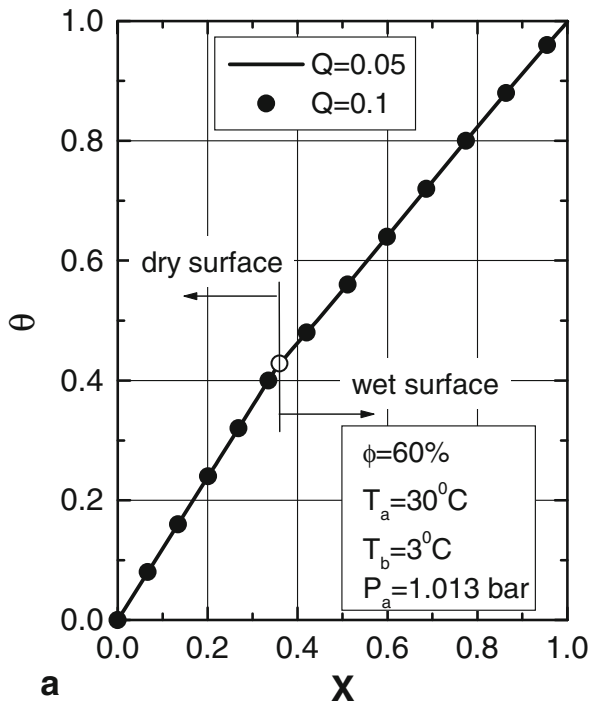

Fig. 4a indicates. The optimum profile obtained from the present analysis for fully wet surface conditions is shown in Fig. 4b. The fin thickness at the tip is zero for the optimum wet fin, as in the case of dry surface fins. An increase in $Q$ increases the envelope fin shape, as expected. The temperature distribution in optimum fins for partially wet surface conditions is depicted in Fig. 5a. A variation of temperature in the dry and wet sections of the fin is clearly exhibited, separated by the dew-point temperature. The temperature gradient in the dry section is greater than that in the wet section. However, the variation of temperature in both the dry and wet sections can be approximated linearly. The optimum fin profile for partially wet fins is also plotted in Fig. 5b for constrained heat transfer duties. The fin profiles for the dry and wet sections are shown separately in this figure. The fin profile for the dry surface zone is not altered significantly by the variation of heat transfer duty $Q$, whereas this variation is predominant in the wet surface zone.

The effect of relative humidity on the optimization parameters of wet fins is shown in Fig. 6. Figure 6a depicts the fin volume as a function of the constrained heat transfer rate for different psychrometric conditions. An increase in the relative humidity of the surrounding air enhances the condensation of moisture on the fin surface, which reduces the envelope shape of the fin for a constant heat duty. Therefore, for any given desired effect, the required fin volume is minimal for fully wet fins. The fin volume for a given relative humidity increases gradually with the heat transfer duty. Figure $6 \mathrm{~b}$ shows the variation of the optimum inverse aspect ratio $\psi_{\text {opt }}$ with respect to the heat duty $\mathrm{Q}$ under different wet surface conditions. For all surface conditions, the geometrical parameter $\psi_{o p t}$ increases as more heat is transferred. Hence, it can be concluded that as the relative 
Fig. 6 Optimum design parameters with respect to the constrained heat transfer rate $Q$ for wet fins: a fin volume; and b $\psi_{\text {opt }}$

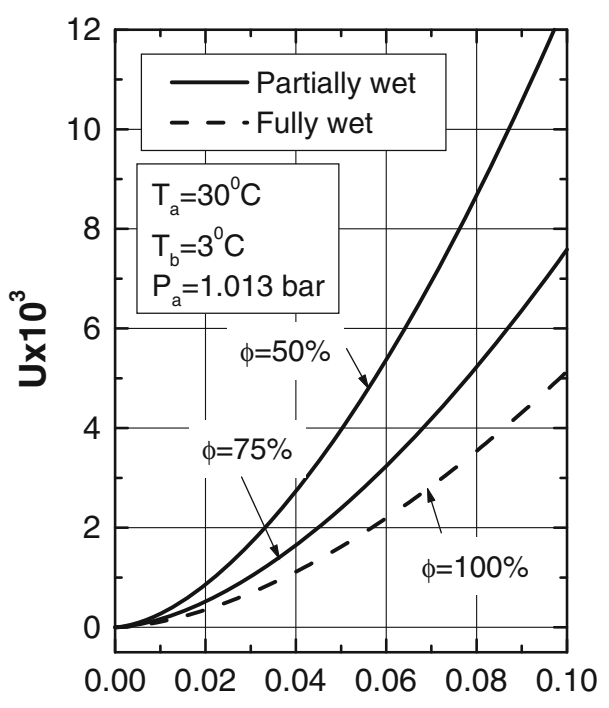

a

Q

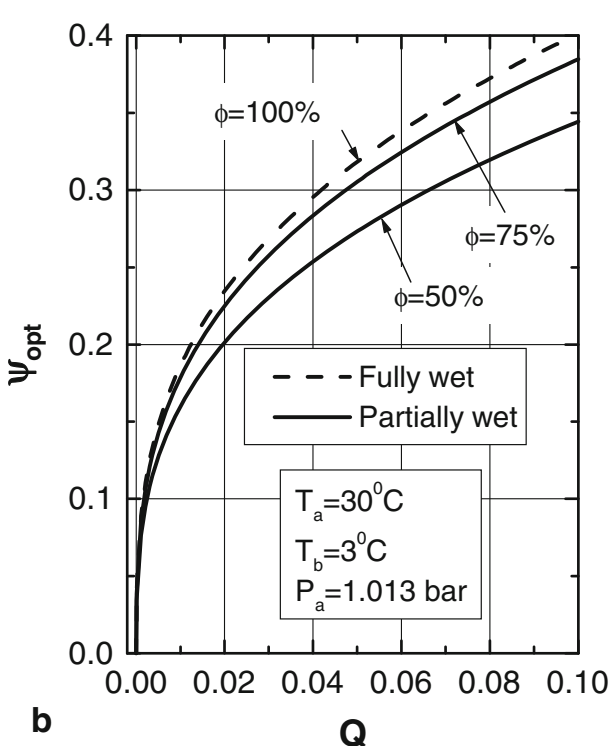

humidity increases, the optimum geometrical parameter $\psi_{\text {opt }}$ also increases when the heat duty remains fixed.

In fin design problems, the performance of dry fins does not depend upon the dry-bulb temperature, base temperature, or atmospheric pressure. On the other hand, these three parameters affect the surface condition of wet fins, and thus influence their performance significantly. The drybulb temperature varies at different locations, and even changes from 1 day to the next. The influence of dry-bulb temperature on fin performance under optimum conditions must therefore be examined. Figure 7 a depicts the optimum profile as a function of dry-bulb temperature for a given design condition. At a constant relative humidity, a higher dry-bulb temperature enhances the moisture content in the air, while at the same time, the latent heat of condensation decreases due to the increasing dew-point temperature. When the dry-bulb temperature increases at a constant relative humidity, condensation of moisture on the fin surface increases, which increases the heat evolved, even though the latent heat decreases. Hence, the envelope shape of the fin profile for optimum wet fins decreases with the dry-bulb temperature at a constant heat transfer rate. In this connection, it should be noted that the design parameter base temperature may be fixed for a particular application. However, for different applications, it may take different values. For example, the fin base temperature of an evaporator coil for domestic refrigerators is different from that for air conditioners. Hence, it may be necessary to study the effect of base temperature variation on the optimum profile shape. For constant dry-bulb temperature and relative humidity, when the base temperature is incremented, the heat transfer rate is reduced as shown in
Fig. 7 Effects of ambient temperature and base temperature on the optimum fin profile: a variation of $T_{a}$; and b variation of $T_{b}$
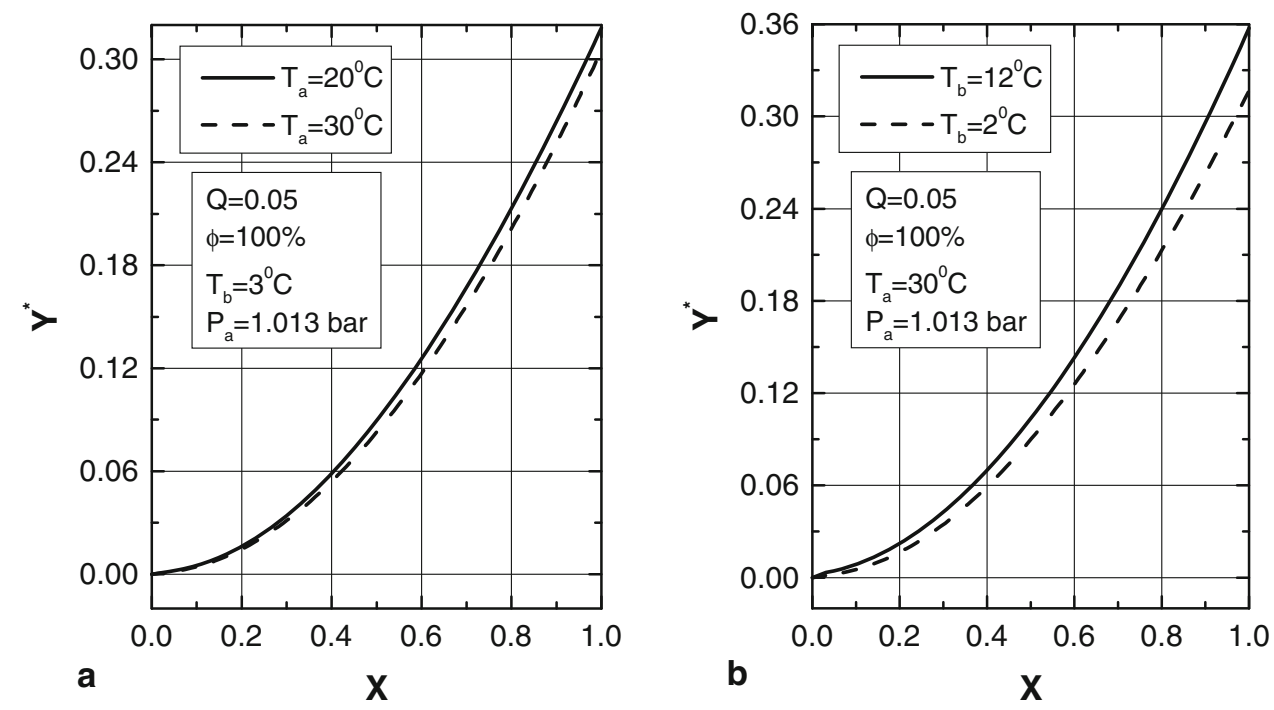
Fig. $7 b$ due to the increased surface temperature of the fin. Since the present optimization study was carried out with a constrained heat transfer duty, a larger fin shape is thus obviously required at the optimum point when the base temperature increases.

\section{Conclusions}

Fin optimization can be accomplished via two approaches. In the first approach, the shape of the fin is optimized either by maximizing the heat transfer rate for a given fin volume, or equivalently by minimizing the fin volume for a desired heat transfer duty. The second approach determines the optimum dimensions for a known fin shape satisfying the optimization conditions. It has already been noted that the former approach is superior with respect to the heat transfer rate per unit volume. In the existing literature, optimization of fins under dry surface conditions has generally been carried out using the first approach. However, in refrigeration, air conditioning, and aerospace applications, the surface temperature of the fins is lower than the dew-point temperature of the surrounding air, and thus the air is dehumidified due to the condensation of moisture on the fin surface. This phenomenon renders the analysis of fins much more complex. In the present study, a methodology was proposed for determining the optimum shape of pin fins under dehumidifying conditions utilizing the first approach to optimization. The analysis presented here is applicable to dry, fully wet, and partially wet surface conditions. The effect of different design conditions on the optimum fin shape was also studied. From the optimization study, it can be inferred that unlike the case of dry surfaces, the optimum conditions for wet fins depend upon the fin base temperature and psychrometric properties of air. However, in every case study of optimum wet fins, the tip temperature equalizes with the surrounding temperature, as with dry fins. The temperature distribution of the optimum wet fin may not be exactly a linear function.

Acknowledgments This work was supported by Brain Korea 21 .

Open Access This article is distributed under the terms of the Creative Commons Attribution License which permits any use, distribution, and reproduction in any medium, provided the original author(s) and the source are credited.

\section{References}

1. O’Brien NG, Tuner RI (1963) Fin thermal efficiency during simultaneous heat and mass transfer. AIChE J 11:546-548
2. Threlkeld JL (1970) Thermal environment engineering. PrenticeHall, New York

3. McQuiston FC (1975) Fin efficiency with combined heat and mass transfer. ASHRAE Trans 71:350-355

4. Kilic A, Onat K (1981) The optimum shape for convecting rectangular fins when condensation occurs. Warme-und Stoffubertragung 15:125-133

5. Coney JER, Sheppard CGW, El-Shafei EAM (1989) Fin performance with condensation from humid air: a numerical investigation. Int J Heat Fluid Flow 10:224-231

6. Wu G, Bong TY (1994) Overall efficiency of a straight fin with combined heat and mass transfer. ASRAE Trans 100(1):365-374

7. Kundu B (2009) Approximate analytic solutions for performance of fully and partially wet-fins with the treatment of cubic polynomial relation between humidity ratio and temperature. Int $\mathbf{J}$ Therm Sci 48:2108-2118

8. Lin YT, Hsu KC, Chang YJ, Wang CC (2001) Performance of rectangular fin in wet conditions: visualization and wet fin efficiency. ASME J Heat Transfer 123:827-836

9. Kundu B (2007) Performance and optimum design analysis of longitudinal and pin fins with simultaneous heat and mass transfer: unified and comparative investigations. Appl Therm Eng 27(5-6):976-987

10. Kundu B (2007) Performance and optimization analysis of SRC profile fins subject to simultaneous heat and mass transfer. Int $\mathrm{J}$ Heat Mass Transfer 50:1645-1655

11. Kundu B (2009) Analysis of thermal performance and optimization of concentric circular fins under dehumidifying conditions. Int J Heat Mass Transfer 52(11-12):2646-2659

12. Kundu B (2002) Analytical study of the effect of dehumidification of air on the performance and optimization of straight tapered fins. Int Comm Heat Mass Transfer 29:269-278

13. Kundu B, Barman D (2011) An analytical prediction for performance and optimization of an annular fin assembly of trapezoidal profile under dehumidifying conditions. Energy 36:2572-2588

14. Toner M, Kilic A, Onat K (1982) Comparison of rectangular and triangular fins when condensation occurs. Warme-und Stoffubertragung 17:65-72

15. Kundu B, Barman D, Debnath S (2008) An analytical approach for predicting fin performance of triangular fins subject to simultaneous heat and mass transfer. Int J Refrig 31:1113-1120

16. Sharqawy MH, Zubair SM (2008) Efficiency and optimization of straight fins with combined heat and mass transfer-an analytical solution. Appl Therm Eng 28:2279-2288

17. Liu CY (1962) A variational problem with application to cooling fins. J Soc Ind Appl Math 10:19-29

18. Liu CY (1961) A variational problem relating to cooling fins with heat generation. Q Appl Math 19:245-251

19. Natarajan U, Shenoy UV (1990) Optimum shapes of convective pin fins with variable heat transfer coefficient. J Franklin Inst 327:965-982

20. Razelos P, Kakatsios X (2000) Optimum dimensions of convecting-radiating fins: part I-longitudinal fin. Appl Therm Eng 20:1161-1192

21. Kundu B, Das PK (2005) Optimum profile of thin fins with volumetric heat generation: a unified approach. ASME J Heat Transfer 126(5):862-868

22. Kundu B (2008) Optimization of fins under wet conditions using variational principle. AIAA $\mathrm{J}$ Thermophys Heat Transfer 22:604-616

23. Kern DQ, Kraus AD (1972) Extended surface heat transfer. McGraw-Hill, New York

24. Scarborough JB (1966) Numerical mathematical analysis. Oxford \& IBH, New Delhi 